\title{
A method for predicting peak work rate for cycle ergometer and treadmill ramp tests
}

Jittima Saengsuwan ${ }^{1,2,3 *}$

Email: jittima.saengsuwan@bfh.ch

Tobias $\mathrm{Nef}^{2}$

Email: tobias.nef@artorg.unibe.ch

Kenneth J. Hunt ${ }^{1}$

Email: kenneth.hunt@bfh.ch

Affiliations:

${ }^{1}$ Institute for Rehabilitation and Performance Technology, Division of Mechanical Engineering, Department of Engineering and Information Technology, Bern University of Applied Sciences, Burgdorf, Switzerland

${ }^{2}$ ARTORG Center for Biomedical Engineering Research, Gerontechnology and Rehabilitation Research Group, University of Bern, Bern, Switzerland

${ }^{3}$ Department of Physical Medicine and Rehabilitation, Faculty of Medicine, Khon Kaen University, Khon Kaen, Thailand

Published as:

Saengsuwan, J., Nef, T., Hunt, K.J. (2017) A method for predicting peak work rate for cycle ergometer and treadmill ramp tests. Clinical Physiology and Functional Imaging. 37:610-614. https://doi: 10.1111/cpf.12344. 


\section{Summary}

Background: Prediction of peak work rate (WRpeak) for incremental exercise testing (IET) is important to bring subjects to their maximal performance within the recommended 8-12 min. This study developed a novel method for prediction of WRpeak for IET on cycles and treadmills.

Methods: Peak metabolic equivalent of task (METpred) was predicted based on an existing nonexercise prediction formula, and then, predicted peak work rate (WRpred) was derived from separate formula for the cycle and treadmill. Eighteen healthy subjects were included.

Results: In males, there was no difference between WRpred versus WRpeak for both the cycle ergometer (277.7 versus $275.6 \mathrm{~W}, \mathrm{p}=0.70$ ) and the treadmill (264.1 versus $260.5, \mathrm{p}=0.58$ ). In females, there was no difference between WRpred versus WRpeak for the cycle ergometer (187.1 versus 188.3 $W, p=0.90$ ), but a statistically significant difference was found between WRpred versus WRpeak on the treadmill (178.6 versus $151.9 \mathrm{~W}, \mathrm{p}<0.05$ ).

For males, the mean absolute percentage errors for WRpred versus WRpeak were $4.6 \%$ and $5.7 \%$ for the cycle and treadmill, respectively. For females, the errors were $12.2 \%$ and $20.8 \%$. The algorithm was successful in achieving the required duration of $8-12 \mathrm{~min}$ in 33 of 36 cases.

Conclusions: The peak work rate prediction protocol was accurate in male subjects for both the cycle and the treadmill. In female subjects, the method was accurate for the cycle, but systematically overpredicted the peak work rate on the treadmill. The protocol requires further adaptation for females on the treadmill. 


\section{Introduction}

Maximal or peak oxygen uptake (maximal $\mathrm{V}^{\prime} \mathrm{O}_{2}$ or peak $\mathrm{V}^{\prime} \mathrm{O}_{2}$ ) obtained from incremental exercise testing (IET) is the gold standard measure for determining cardiopulmonary fitness (Pescatello et al., 2014). This test is usually done on a treadmill or a cycle ergometer. It is recommended that the work rate increment for a ramp protocol should be chosen so that the total IET time lies in the range of 8 12 min to improve subjects' tolerance and test quality (Buchfuhrer et al., 1983; Myers et al., 1992; Myers \& Froelicher, 1993; Pescatello et al., 2014). A shorter exercise test duration of less than 8 min results in approximately $10 \%$ reduction of maximal $\mathrm{V}^{\prime} \mathrm{O}_{2}$ and produces a nonlinear relationship between oxygen uptake $\left(V^{\prime} \mathrm{O}_{2}\right.$ ) and work rate (WR) (Buchfuhrer et al., 1983; Balady et al., 2010). A longer test (more than $17 \mathrm{~min}$ ) results in approximately $5 \%$ reduction of maximal $\mathrm{V}^{\prime} \mathrm{O}_{2}$ with the longest of these tests having the greatest reduction (Buchfuhrer et al., 1983). Subjects found longer tests more stressful: they complained about seat discomfort with the long cycle tests and low back pain with the long treadmill tests (Buchfuhrer et al., 1983). Additionally, subjects may terminate the tests because of muscle fatigue or orthopaedic factors rather than cardiopulmonary end points (Balady et al., 2010). Thus, long tests waste time, increase the physiological and psychological stress and may distort the results (Buchfuhrer et al., 1983).

The accurate prediction of peak work rate is important to bring the subjects to their maximal performance within a desirable time. Subjects usually need to perform a short maximal test or a warm up test to predict the peak work rate before an IET, which is not convenient to implement in daily practice (Arts et al., 1993; Loe et al., 2014; Myers et al., 1992). Recently, there was an attempt to use non-exercise prediction of maximal $\mathrm{V}^{\prime} \mathrm{O}_{2}$ to design IET protocols (Maeder et al., 2006; da Silva et al., 2012; Cunha et al., 2015). For example, a recently published study by Cunha et al. (2015) used a non-exercise model (Matthews et al., 1999) and ACSM's metabolic equations (Thompson et al., 2009) to determine the cycle and treadmill ramp protocol and found promising results.

In this work, we developed formulae to predict the peak work rate on a cycle ergometer and a treadmill from the predicted maximal $\mathrm{V}^{\prime} \mathrm{O}_{2}$. The maximal $\mathrm{V}^{\prime} \mathrm{O}_{2}$ was calculated from an existing nonexercise prediction formula which is based on regression analysis of data from the National Aeronautics and Space Administration/Johnson Space Center (NASA) (Jurca et al., 2005). This formula is easy to administer (no nomogram needed) and provides the exact value of the predicted metabolic equivalent of task (MET). The formula from the NASA model shows a high model fit with minimal standard error of estimates and has one of the highest model fits among other existing non-exercise prediction models (Wisen et al., 2002; Jurca et al., 2005; Maeder et al., 2005; Nes et al., 2011).

The ramp protocol has the advantage of stressing the subject for only a few minutes at high work rates and providing a favourable effect on gas exchange dynamics (Myers et al., 1991; Wasserman et al., 1999). There are many existing ramp protocols for the treadmill. Cunha et al. (2015) used a ramp protocol with a treadmill speed of $4 \mathrm{~km} / \mathrm{h}$ at the beginning and $6 \mathrm{~km} / \mathrm{h}$ at the end. The average slope at the end of the exercise was high (22\%). Porszasz et al. (2003) implemented a protocol using a linear increase in speed and nonlinear increase in angle to achieve a linear work rate ramp; however, this resulted in a high final treadmill slope in lighter subjects (approximately $20 \%$ ). Here, we implemented a 
protocol combining simultaneous nonlinear changes in speed and angle at the same relative proportion (Hunt, 2008).

The aim of this work was to develop and evaluate a novel method for prediction of peak work rate for IET on both a cycle ergometer and a treadmill. This allows the prediction of individual peak work rate which does not require a pre-test evaluation.

\section{Methods}

\section{Subjects}

This study was reviewed and approved by the Ethics Review Committee of the Swiss Canton of Bern, Switzerland (Ref.002/12). All research subjects gave their written informed consent before participating in the study.

18 healthy subjects (10 male) participated (Table 1). Subjects were aged 18-50 years and had no cardiovascular, pulmonary or musculoskeletal disease that might have interfered with the exercise testing.

Table 1. Demographic data of subjects.

\begin{tabular}{lcc}
\hline Characteristic & Male $(\mathbf{n}=\mathbf{1 0})$ & Female $(\mathbf{n}=\mathbf{8})$ \\
\hline Age $[$ years] & $30(7.5)$ & $26.8(4.1)$ \\
Height $[\mathrm{cm}]$ & $177(9.3)$ & $166.8(7.7)$ \\
Body mass $[\mathrm{kg}]$ & $75.0(11.9)$ & $61.8(10.1)$ \\
Body mass index $\left[\mathrm{kg} / \mathrm{m}^{2}\right]$ & $23.2(2.3)$ & $22.1(2.0)$ \\
Activity level* & $3.4(0.8)$ & $3.4(1.4)$ \\
\hline
\end{tabular}

Values are: mean (standard deviation)

*Activity level was calculated according to Jurca et al. (2005). In brief, level 1 refers to inactive or little activity, level 2 refers to a regular $\geq 5$ days/week) low level of exertion (10 $\mathrm{min}$ ), level 3 refers to aerobic exercise of 20-60 minweek, level 4 refers to aerobic exercise of 1-3 hweek, and level 5 refers to aerobic exercise of $>3$ hweek.

\section{Prediction of peak Metabolic Equivalent of Task (METpred) and predicted peak work rate (WRpred)}

To estimate the predicted peak work rate (WRpred) for each individual, we first predicted the peak metabolic equivalent of task (METpred). This formula was derived from regression analysis of data 
from the National Aeronautics and Space Administration/Johnson Space Center (NASA) (Jurca et al., 2005) which were from 1458 men and 405 women aged 20-70 years who did the exercise testing on a treadmill (Jackson et al., 1995, 1996). Here, the METpred was calculated from gender, age, body mass index, resting heart rate and self-reported physical activity levels (Jurca et al., 2005).

WRpred was derived from predicted maximal $\mathrm{V}^{\prime} \mathrm{O}_{2}$ minus the baseline $\mathrm{V}^{\prime} \mathrm{O}_{2}$ during walking/cycling before the start of IET divided by the slope of the oxygen uptake $\left(\mathrm{V}^{\prime} \mathrm{O}_{2}\right)$-work rate $(\mathrm{WR})$ relationship:

$$
\text { WRpred }=\frac{\left(\left(\frac{\text { METpred }}{1.1}\right)-1.8\right) \cdot 3.5 \cdot \text { mass }}{9.6}
$$

for the cycle ergometer, and

$$
\text { WRpred }=\frac{(\text { METpred }-1.8) \cdot 3.5 \cdot \text { mass }}{11.4}
$$

For the treadmill.

The factor 3.5 appears because $1 \mathrm{MET}$ is equivalent to an oxygen uptake $\left(\mathrm{V}^{\prime} \mathrm{O}_{2}\right)$ of $3.5 \mathrm{~mL} / \mathrm{kg} / \mathrm{min}$ (Pescatello et al., 2014) while 1.8 is the baseline MET during walking or unloaded cycling measured in our pilot data. The numbers 9.6 and $11.4 \mathrm{ml} / \mathrm{min} / \mathrm{W}$ are the slopes of the $\mathrm{V}^{\prime} \mathrm{O}_{2}-\mathrm{WR}$ relationship found for the cycle and treadmill ramp tests established by Porszasz et al. (2003). We divided METpred by 1.1 for the prediction of maximal $\mathrm{V}^{\prime} \mathrm{O}_{2}$ on the cycle ergometer because maximal $\mathrm{V}^{\prime} \mathrm{O}_{2}$ achieved by the cycle ergometer is generally approximately $10 \%$ lower than the treadmill (Saengsuwan et al., 2015) and this factor was used by Wasserman et al. (1999) for the translation of maximal $\mathrm{V}^{\prime} \mathrm{O}_{2}$ from the treadmill to the cycle ergometer.

\section{Incremental exercise tests}

Each subject performed 2 tests on the cycle ergometer and 2 tests on the treadmill. The test sequence for each subject was computer randomized, and each test was at least 48 hours apart. The first test on each device was a familiarization and the data from the second test on each device were used for the analysis. Subjects were instructed to avoid intense physical activity 24 hours before each test and not to consume caffeine and nicotine at least 3 hours before each test (Pina et al., 1995). Before each test the subject had 5 min of warm up on either the cycle ergometer or the treadmill.

Work rate slope was calculated to bring the subject to their predicted peak in $10 \mathrm{~min}$, i.e. slope = WRpred/10 [W/min].

\section{Cycle ergometer}

The ramp test was carried out on an electronically controlled cycle ergometer (LC7; Monark Exercise $A B$, Sweden). The protocol consisted of 3 min of rest, 3 min of unloaded cycling, and then the work rate was increased at the rate of $12-40 \mathrm{~W} / \mathrm{min}$ (i.e., the individualised slope) until the subject reached exhaustion. A recovery period of unloaded cycling of $3 \mathrm{~min}$ then followed. 


\section{Treadmill}

A linear increase in work rate for the treadmill ramp protocol was implemented using simultaneous nonlinear changes in both speed and slope. Here, the individual's predicted work rate was used to calculate the speed and slope for each 30-s period on the treadmill (Venus, h/p/cosmos GmbH, Germany) during the ramp test. The protocol consisted of $3 \mathrm{~min}$ of rest, $3 \mathrm{~min}$ of walking at $0.9 \mathrm{~km} / \mathrm{h}$, then the ramp phase where work rate was increased at the individualised rate of $14-30 \mathrm{~W} / \mathrm{min}$ until the subject reached exhaustion. A recovery period of walking at $2 \mathrm{~km} / \mathrm{h}$ for $3 \mathrm{~min}$ followed.

\section{Outcome measures}

The main outcomes were the predicted and peak work rates (WRpred versus WRpeak) for both the treadmill and the cycle ergometer. The treadmill work rate was calculated with the formula WR = mass.g.v. $\sin (\theta)$, where $v$ is treadmill speed and $\theta$ is treadmill angle. The secondary outcomes were incremental exercise duration, the highest treadmill speed and slope and the number of subjects who reached their peak work rate within 8-12 min.

\section{Statistical analyses}

Agreement between the predicted work rate (WRpred) and the peak work rate (WRpeak) was analysed using paired t-tests and the Bland and Altman method (Bland \& Altman, 1986). The absolute error was calculated as |WRpred - WRpeak|. The absolute percentage error was calculated from (absolute error/WRpeak)·100\%. All analyses were performed using SPSS (version 19.0, IBM Corp, Armonk, NY, USA).

\section{Results}

Mean values of the predicted work rate (WRpred) and peak work rate (WRpeak) were similar for the cycle ergometer ( 237.4 versus $236.8 \mathrm{~W}, \mathrm{p}=0.90$ ). For the treadmill, the mean predicted work rate was higher than the observation (226.1 versus $212.3 \mathrm{~W}, \mathrm{p}<0.05$ ) (Table 2 ). 
Table 2. Accuracy of the predicted work rate (WRpred) in all subjects and classified by gender.

\begin{tabular}{|c|c|c|c|c|c|c|c|}
\hline & $\begin{array}{c}\text { Mean } \\
\text { WRpred } \\
\text { (W) }\end{array}$ & $\begin{array}{c}\text { Mean } \\
\text { WRpeak } \\
\text { (W) }\end{array}$ & $\begin{array}{c}\mathrm{p} \text { - } \\
\text { value }\end{array}$ & $\begin{array}{c}\text { Mean } \\
\text { difference } \\
\text { (W) }\end{array}$ & $\begin{array}{c}95 \% \text { LoA } \\
\text { W) }\end{array}$ & $\begin{array}{c}\text { Mean } \\
\text { Absolute } \\
\text { Error }(W)\end{array}$ & $\begin{array}{c}\text { Mean } \\
\text { Absolute } \\
\text { Percentage } \\
\text { Error (\%) }\end{array}$ \\
\hline \multicolumn{8}{|l|}{ Total } \\
\hline Cycle & 237.4 & 236.8 & 0.90 & 0.6 & -37.6 to 38.8 & 15.1 & 7.9 \\
\hline Treadmill & 226.1 & 212.3 & 0.02 & 13.8 & -31.7 to 59.3 & 20.9 & 12.4 \\
\hline \multicolumn{8}{|l|}{ Male } \\
\hline Cycle & 277.7 & 275.6 & 0.70 & 2.1 & -28.3 to 32.5 & 11.5 & 4.6 \\
\hline Treadmill & 264.1 & 260.5 & 0.58 & 3.6 & -34.7 to 41.8 & 14.0 & 5.7 \\
\hline \multicolumn{8}{|l|}{ Female } \\
\hline Cycle & 187.1 & 188.3 & 0.90 & -1.2 & -49.5 to 47.1 & 19.7 & 12.2 \\
\hline Treadmill & 178.6 & 151.9 & 0.01 & 26.7 & -16.4 to 69.8 & 29.5 & 20.8 \\
\hline
\end{tabular}

Stratifying the outcomes by gender revealed that there was no statistically significant difference between WRpred versus WRpeak for both the cycle ergometer ( 277.7 versus $275.6 \mathrm{~W}, \mathrm{p}=0.70$ ) and the treadmill (264.1 versus $260.5, p=0.58$ ) in males. Additionally, there was no difference between WRpred versus WRpeak for the cycle ergometer (187.1 versus $188.3 \mathrm{~W}, \mathrm{p}=0.90$ ) in females. However, a statistically significant difference was found between WRpred versus WRpeak in females on the treadmill (178.6 versus $151.9 \mathrm{~W}, \mathrm{p}<0.05$ ). The overall difference seen on the treadmill therefore stems from the female group.

The $95 \%$ limits of agreement (LoA) for the cycle ergometer WRpred and WRpeak difference were -28.3 to $32.5 \mathrm{~W}$ in males and -49.5 to $47.1 \mathrm{~W}$ in females. The $95 \%$ LoA for the treadmill WRpred and WRpeak difference were -34.7 to $41.8 \mathrm{~W}$ in males and -16.4 to $69.8 \mathrm{~W}$ in females.

For males, the mean absolute percentage errors were $4.6 \%$ and $5.7 \%$ for the cycle and treadmill, respectively. For females, the mean absolute percentage errors were $12.2 \%$ and $20.8 \%$ for the cycle and treadmill, respectively.

Maximal work rate was reached, on average, in $9 \min 47 \mathrm{~s}(9 \min 44 \mathrm{~s}$ in males and $9 \min 51 \mathrm{~s}$ in females) and $9 \min 24 \mathrm{~s}$ ( 9 min $54 \mathrm{~s}$ in males and $8 \mathrm{~min} 24 \mathrm{~s}$ in females) for the cycle and treadmill, respectively. The peak treadmill speed and slope were $8.2 \mathrm{~km} / \mathrm{h}$ and $15.6 \%$ for males, and $7.5 \mathrm{~km} / \mathrm{h}$ and 
$12.2 \%$ for females. All 18 subjects reached their maximal performance in 8-12 min on the cycle ergometer. Fifteen of the 18 subjects (83.3\%) reached maximal performance in $8-12$ min on the treadmill, but three females reached their maximal performance before $8 \mathrm{~min}$ ( 1 subject reached it at $7 \mathrm{~min} 30 \mathrm{~s}$ and 2 subjects reached it at $7 \mathrm{~min} 50 \mathrm{~s}$ ).

\section{Discussion}

The aim of this work was to develop and evaluate a novel method for prediction of peak work rate for IET on both a cycle ergometer and a treadmill.

The first main finding from the study was that WRpeak can be accurately predicted for males with an error of less than $6 \%$ for both the cycle ergometer and the treadmill. However, the WRpeak prediction for females had a higher error (12.2\% for the cycle ergometer and $20.8 \%$ for the treadmill). The second main finding was that the implementation of the prediction of WRpeak for individualized exercise protocol resulted in $100 \%$ of subjects reaching their maximal performance in 8-12 min on the cycle ergometer and $83 \%$ on the treadmill, that is 33 of 36 cases overall $(92 \%)$.

Overall, the WRpeak prediction for the cycle ergometer was more accurate than the treadmill. The WRpeak prediction for the cycle ergometer in this study (mean difference between WRpred and WRpeak was $0.6 \mathrm{~W}$ and $95 \%$ LoA was -37.6 to $38.8 \mathrm{~W}$ ) was favourably comparable to the study of Cunha et al. (2015), which used a different non-exercise prediction model and the ACSM's metabolic equations to determine the cycle work rate, and found that the mean difference between WRpred and WRpeak was $-1 \mathrm{~W}$ and $95 \%$ LoA was -54.1 to $52.1 \mathrm{~W}$.

The work rate on the treadmill is more difficult to correctly quantify because there are unmeasured sources of work, i.e. the baseline work while running at zero slope, which increases as treadmill speed increases (Porszasz et al., 2003). This may account for the lower accuracy of the WRpeak prediction on the treadmill. Additionally, the lower accuracy for females may be because of the higher energy expenditure needed for the same work rate in females compared to males (Abadi et al., 2010). This may be the result of biomechanical differences. It was found in the study of van der Walt \& Wyndham (1973) that leg length has a negative correlation with oxygen uptake. Furthermore, it was found that at the same running velocity, females have a shorter stride length and higher stride rate compared to males (Eiiliot \& Blanksby, 1979).

The prediction of WRpeak and the integration with a protocol that includes simultaneous increases in speed and angle resulted in a lower slope at the time of the subjects' maximal performance. In the study of Porszasz et al. (2003), which used a linear increase in speed and nonlinear increase in angle to achieve a linear work rate ramp, it was shown that lighter subjects had a final inclination as high as $20 \%$. In our study, as the speed and slope were equally changed, the maximal treadmill slope never reached $18 \%$ in any subject. Moreover, with the use of simultaneous nonlinear changes in speed and slope, one can balance the speed and slope changes arbitrarily. 
This study has some limitations that should be addressed. First of all, the formulae can only be used in healthy subjects because we have implemented formulae to predict WRpeak from the $\mathrm{V}^{\prime} \mathrm{O}_{2}-\mathrm{WR}$ relationship from normal subject data (Porszasz et al., 2003). In some patient populations such as those with heart disease, it is known that the $\mathrm{V}^{\prime} \mathrm{O}_{2}-\mathrm{WR}$ relationship will be lower than normal subjects during exercise, thus limiting the usability of these formulae (Myers et al., 2015). Further modification of the formulae to predict WRpeak for the treadmill in females is necessary, and a validation study with a higher number of participants and wider level of fitness status should be carried out in the future.

\section{Conclusion}

The peak work rate prediction protocol was accurate in male subjects for both the cycle and the treadmill. In female subjects, the method was accurate for the cycle but systematically over-predicted the peak work rate on the treadmill. The protocol requires further adaptation and experimental investigation for females on the treadmill.

\section{Acknowledgement}

We thank Marco Laubacher (Bern University of Applied Sciences) for contributions to development of the overall study protocol. 


\section{References}

Abadi FH, Muhamad TA, Salamuddin N. Energy expenditure through walking: meta analysis on gender and age. Procedia Soc Behav Sci (2010); 7: 512-521.

Arts FJ, Kuipers H, Jeukendrup AE, Saris WH. A short cycle ergometer test to predict maximal workload and maximal oxygen uptake. Int J Sports Med (1993); 14: 460-464.

Balady GJ, Arena R, Sietsema K, Myers J, Coke L, Fletcher GF, Forman D, Franklin B, Guazzi M, Gulati M, Keteyian SJ, Lavie CJ, Macko R, Mancini D, Milani RV. Clinician's guide to cardiopulmonary exercise testing in adults: a scientific statement from the American Heart Association. Circulation (2010); 122: 191-225.

Bland JM, Altman DG. Statistical methods for assessing agreement between two methods of clinical measurement. Lancet (1986); 1: 307-310.

Buchfuhrer MJ, Hansen JE, Robinson TE, Sue DY, Wasserman K, Whipp BJ. Optimizing the exercise protocol for cardiopulmonary assessment. J Appl Physiol Respir Environ Exerc Physiol (1983); 55: 1558-1564.

Cunha FA, Midgley A, Montenegro R, Vasconcellos F, Farinatti P. Utility of a non-exercise VO2max prediction model for designing ramp test protocols. Int J Sports Med (2015); 36: 796-802.

Hunt KJ. Treadmill control protocols for arbitrary work rate profiles combining simultaneous nonlinear changes in speed and angle. Biomed Signal Process Control (2008); 3: 278-282.

Jackson AS, Beard EF, Wier LT, Ross RM, Stuteville JE, Blair SN. Changes in aerobic power of men, ages 25-70 yr. Med Sci Sports Exerc (1995); 27: 113-120.

Jackson AS, Wier LT, Ayers GW, Beard EF, Stuteville JE, Blair SN. Changes in aerobic power of women, ages 20-64 yr. Med Sci Sports Exerc (1996); 28: 884-891.

Jurca R, Jackson AS, LaMonte MJ, Morrow JR Jr, Blair SN, Wareham NJ, Haskell WL, van Mechelen W, Church TS, Jakicic JM, Laukkanen R. Assessing cardiorespiratory fitness without performing exercise testing. Am J Prev Med (2005); 29: 185-193.

Loe H, Steinshamn S, Wisloff U. Cardio-respiratory reference data in 4631 healthy men and women 20-90 years: The HUNT 3 fitness study. PLoS One (2014); 9: e113884.

Maeder M, Wolber T, Atefy R, Gadza M, Ammann P, Myers J, Rickli H. A nomogram to select the optimal treadmill ramp protocol in subjects with high exercise capacity: validation and comparison with the Bruce protocol. I Cardiopulm Rehabil (2006); 26: 16-23.

Maeder M, Wolber T, Atefy R, Gadza M, Ammann P, Myers J, Rickli H. Impact of the exercise mode on exercise capacity: bicycle testing revisited. Chest (2005); 128: 2804-2811.

Matthews CE, Heil DP, Freedson PS, Pastides H. Classification of cardiorespiratory fitness without exercise testing. Med Sci Sports Exerc (1999); 31: 486-493.

Myers J, Arena R, Cahalin LP, Labate V, Guazzi M. Cardiopulmonary exercise testing in heart failure. Curr Probl Cardiol (2015); 40: 322-372.

Myers J, Buchanan N, Smith D, Neutel J, Bowes E, Walsh D, Froelicher VF. Individualized ramp treadmill. Observations on a new protocol. Chest (1992); 101: S236-S241.

Myers J, Buchanan N, Walsh D, Kraemer M, McAuley P, Hamilton-Wessler M, Froelicher VF. Comparison of the ramp versus standard exercise protocols. J Am Coll Cardiol (1991); 17: 1334-1342.

Myers J, Froelicher VF. Exercise testing. Procedures and implementation. Cardiol Clin (1993); 11: 199213.

Nes BM, Janszky I, Vatten L, Nilsen TI, Aspenes ST, Wisloff U. Estimating VO2peak from a nonexercise prediction model: the HUNT Study, Norway. Med Sci Sports Exerc (2011); 43: 2024-2030. 
Pescatello LS, Arena R, Riebe D, Thompson PD, eds. ACSM's guidelines for exercise testing and prescription (2014). 9th ed. Philadelphia: Lippincott Williams \& Wilkins.

Pina IL, Balady GJ, Hanson P, Labovitz AJ, Madonna DW, Myers J. Guidelines for clinical exercise testing laboratories. A statement for healthcare professionals from the Committee on Exercise and Cardiac Rehabilitation, American Heart Association. Circulation (1995); 91: 912 921.

Porszasz J, Casaburi R, Somfay A, Woodhouse LJ, Whipp BJ. A treadmill ramp protocol using simultaneous changes in speed and grade. Med Sci Sports Exerc (2003); 35: 1596-1603.

Saengsuwan J, Nef T, Laubacher M, Hunt KJ. Comparison of peak cardiopulmonary performance parameters on a robotics-assisted tilt table, a cycle and a treadmill. PLoS One (2015); 10 e0122767.

da Silva SC, Monteiro WD, Cunha FA, Myers J, Farinatti PT. Determination of best criteria to determine final and initial speeds within ramp exercise testing protocols. Pulm Med (2012);

2012: 542402 .

Thompson WR, Gordon NF, Pescatello LS, eds. ACSM's guidelines for exercise testing and prescription (2009). 8th ed. Philadelphia: Lippincott Williams \& Wilkins.

van der Walt WH, Wyndham $\mathrm{CH}$. An equation for prediction of energy expenditure of walking and running. J Appl Physiol (1973); 34: 559-563.

Wasserman K, Hansen JE, Sue DY, Casaburi R, Whipp BJ. Principles of exercise testing and interpretation (1999). 3rd ed. Philadelphia: Lippincott Williams \& Wilkins.

Wisen AG, Farazdaghi RG, Wohlfart B. A novel rating scale to predict maximal exercise capacity. Eur J Appl Physiol (2002); 87: 350-357. 